\title{
Initial Test Results of an Ionization Chamber Shower Detector for a LHC Luminosity Monitor
}

\author{
P. Datte, J-F. Beche, M. Haguenauer, P.F. Manfredi, M. Manghisoni, J. Millaud, M. Placidi, L. Ratti, \\ V. Riot, H. Schmickler, V. Speziali and W. Turner
}

\begin{abstract}
A novel segmented, multi-gap, pressurized gas ionization chamber is being developed for optimization of the luminosity of the LHC. The ionization chambers are to be installed in the front quadrupole and zero degree neutral particle absorbers in the high luminosity IRs and sample the energy deposited near the maxima of the hadronic/electromagnetic showers in these absorbers. The ionization chambers are instrumented with low noise, fast, pulse shaping electronics to be capable of resolving individual bunch crossings at $40 \mathrm{MHz}$. In this paper we report the initial results of our second test of this instrumentation in an SPS external proton beam. Single $300 \mathrm{GeV}$ protons are used to simulate the hadronic/electromagnetic showers produced by the forward collision products from the interaction regions of the LHC. The capability of instrumentation to measure the luminosity of individual bunches in a $40 \mathrm{MHz}$ bunch train is demonstrated.
\end{abstract}

Index Terms-Shower detector, Ionization detector, gas monitor.

\section{INTRODUCTION}

$\mathrm{T}$ HE feasibility of utilizing the very forward flux of secondary particles from IP interactions that are intercepted by the neutral particle and front quadrupole absorbers (TAN and TAS) of the LHC for the bunch-by-

Manuscript received: November 7, 2001. This work was supported by the Director, Office of Energy Research, Office of High Energy and Nuclear Physics, Division of High Energy Physics, of the U.S. Dept. of Energy under Contract No. DE-AC03-76SF00098.

J. F. Beche is with the Lawrence Berkeley National Laboratory, Berkeley, CA 94720 USA (telephone: 510-486-4164, e-mail: JFBeche@lbl.gov).

P. S. Datte is with the Lawrence Berkeley National Laboratory, Berkeley, CA 94720 USA (telephone: 510-486-2906, e-mail: PSDatte@lbl.gov), Corresponding author.

M. Haguenauer is with Ecole Polytechnique, F-91128 Palaiseau Cedex 09 France, (telephone: 412276 77198, e-mail: Maurice.Haguenauer@cern.ch).

P. F. Manfredi is with the Lawrence Berkeley National Laboratory, Berkeley, CA 94720 USA (telephone: 510-486-7786, e-mail: PFManfredi@lbl.gov).

M. Manghisoni is with the University of Pavia - INFN Pavia, Pavia, Italy (telephone: 390382 505.222, email: M.manghisoni@ele.unipv.it).

J. E. Millaud is with the Lawrence Berkeley National Laboratory, Berkeley, CA 94720 USA (telephone

L. Ratti is with the University of Pavia - INFN Pavia, Pavia, Italy (telephone: 39 0382 505.222, e-mail: 1.ratti@ele.unipv.it).

V. J. Riot is with the Lawrence Berkeley National Laboratory, Berkeley, CA 94720 USA (telephone: 510-486-2704, e-mail: VJRiot@lbl.gov).

H. Schmickler is with CERN, CH-1211, Geneva 23 Switzerland (telephone number: 412276 77078, e-mail: Hermann.Schmickler@cern.ch).

V. Speziali is with the University of Pavia - INFN Pavia, Pavia, Italy (telephone: 390382 505.216, e-mail: speziali@ele.unipv.it).

W. C. Turner is with the Lawrence Berkeley National Laboratory, Berkeley, CA 94720 USA (telephone: 510-486-7385, e-mail: WCTurner@lbl.gov). bunch measurement and optimization of luminosity has been discussed in previous reports.[1-4] The TAN and TAS absorbers have also been described previously.[3] It is proposed to instrument the neutral particle and front quadrupole absorbers with fast, low noise detectors that sample the energy deposited in the showers produced by the interaction products from the IP. A pressurized, segmented, multi-gap gas ionization chamber coupled with a radiation hard cable to a low noise, cold termination bipolar transistor preamplifier and pulse shaper have been chosen to meet the constraints imposed by radiation, bandwidth and signal to noise ratio. $[2,4]$ The proposed system provides a fast relative luminosity monitor that can be periodically calibrated either by simultaneous measurement of the beam emittance and bunch intensity or against another absolute measurement of luminosity. The advantages of the proposed technique are that it is fast and it is technologically relatively simple to implement. A $1 \%$ measurement of the luminosity of each of the 2835 colliding bunch pairs of the LHC can be carried out in $~ 2000$ turns. The instrumentation would be used as a storage ring operations tool to keep the LHC operating near optimum luminosity. The first beam test results showed sensitivity to showers initiated by single protons, linearity of signal with ionization chamber pressure and location of shower maximum at the anticipated thickness of absorber $(\sim 20 \mathrm{~cm}$ of iron) [6]. Two issues were identified which required further development: (1) reduction of capacitive noise coupling between quadrants and (2) reduction of pulse width and improvement of pulse shape. Capacitive coupling has been eliminated with ground planes between quadrants. The pulse width has been reduced and the pulse shape improved by careful impedance matching of the amplifier to the input cable and reduction of parasitic capacitance and inductance of the ionization chamber electrical connections. In this report we describe the initial results of the second beam test utilizing single $300 \mathrm{GeV}$ protons to initiate showers. The following sections of the report describe; (1) the experimental equipment, (2) the simulations of the hadronic/electro-magnetic showers, (3) the experimental results and the comparison with data from the previous beam test and (4) an analysis of the $40 \mathrm{MHz}$ capability of the instrument. The report ends with a section on conclusions. A companion paper describes the analog electronics.[7]. 


\section{DESCRIPTION OF EXPERIMENTAL EQUIPMENT}

\section{A. Ionization chamber}

A schematic of the ionization chamber is shown in Fig. 1. The ionization chamber is segmented into quadrants; each quadrant consists of sixty $0.5 \mathrm{~mm}$ gaps separated by $1.0 \mathrm{~mm}$ thick $\mathrm{Cu}$ plates.

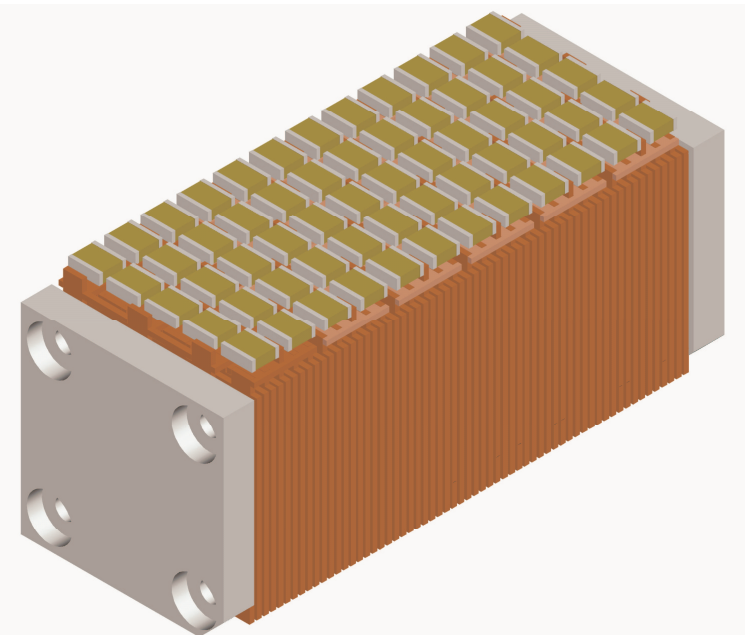

Fig. 1: Schematic of a quadrant of the ionization chamber.

The $0.5 \mathrm{~mm}$ gap width has been chosen so that the sum of the time for ionization electrons to drift across the gap and the amplifier shaping time is less than the $25 \mathrm{nsec}$ bunch spacing of the LHC. The active area of the plates is $40 \mathrm{~mm} x$ $40 \mathrm{~mm}$ and is constrained by the requirement of fitting between the two beam tubes in the neutral particle absorbers of the high luminosity insertions of LHC. The stack of plates in each quadrant is connected electrically 10 in parallel, 6 in series to achieve an equivalent detector capacitance $C_{d} \sim$ $50 \mathrm{pF}$. The precise value of the detector capacitance is chosen by consideration of signal to noise ratio of the combined system including the detector capacitance + cable $\left(\mathrm{R}_{0}=\right.$ $50 \mathrm{Ohms}, \sim 10 \mathrm{nsec}$ delay $)+$ amplifier $(\tau=2.5 \mathrm{nsec}$ shaping time).[5] In order that the connecting cable does not degrade the signal to noise ratio of the system it is necessary to choose the ratio of shaping time to detector rise time so that $\tau / R_{0} C_{d} \sim$ 1 , thus leading to $C_{d} \sim 50 \mathrm{pF}$. The signal to noise ratio of the detector depends linearly on the gas pressure and is less sensitive to other parameters of the system. For this reason the ionization chamber is to be pressurized with up to 10 atmospheres of $\mathrm{Ar}+2 \% \mathrm{~N}_{2}$. For the tests described in this paper data were taken with two quadrants or a single quadrant. With a single quadrant and no ground isolation planes the effective detector capacitance was measured to be $60 \mathrm{pF}$. With two quadrants separated by ground isolation planes the effective detector capacitance was measured to be 150-200pF. The gap between the ground isolation planes and the edges of the ionization chamber is $1 \mathrm{~mm}$. The effective inductance of a single quadrant is calculated to be $<60 \mathrm{nH}$.

\section{B. Setup in a $300 \mathrm{GeV}$ proton beam}

Fig. [2] shows a schematic of the experimental setup in the SPS H4 area at CERN. A low intensity beam of $300 \mathrm{GeV}$ protons is incident on an array of scintillation counters and wire chambers that characterize the beam followed by a variable thickness absorber consisting of $(25 \mathrm{~cm} \times 25 \mathrm{~cm})$, $2.5 \mathrm{~cm}$ thick $\mathrm{Fe}$ plates and the ionization chamber. The distance from the scintillation counters to the front of the absorber was $8.5 \mathrm{~m}$.
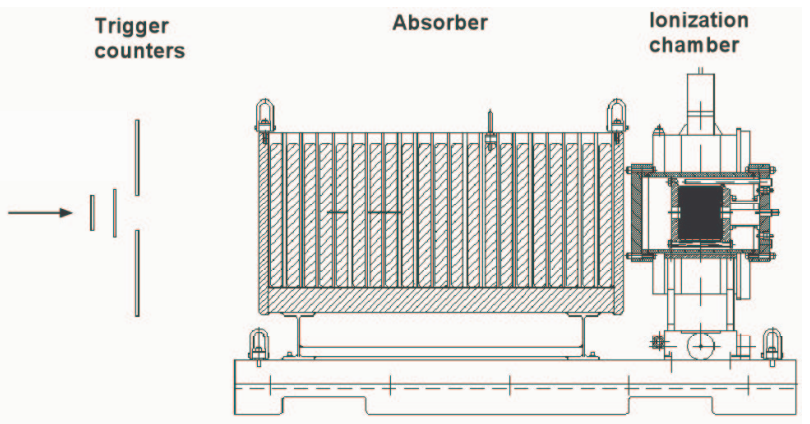

Fig. 2: Schematic of the experimental setup that includes the trigger counters, absorbers and ionization chamber.

The ionization chamber detects the ionizing particles of the hadronic/electromagnetic showers exiting the absorber. The SPS was operated with a 5.2sec slow extraction spill repeated every $15.8 \mathrm{sec}$. The extracted primary protons struck a target to produce the secondary $300 \mathrm{GeV}$ secondary protons of the $\mathrm{H} 4$ beam. The intensity of the beam was typically (3-6 $x 10^{5}$ ) protons per spill. Data acquisition of the ionization chamber pulse was triggered by a coincidence between the $(5 \mathrm{~mm} \times 5 \mathrm{~mm})$ and $(50 \mathrm{~mm} \times 50 \mathrm{~mm})$ scintillation counters.

\section{SIMULATIONS}

The MARS code is used to simulate hadronic/electromagnetic showers that are the basis for estimating the anticipated signal. It is a property of these showers that the shower energy is carried predominantly by hadrons while the energy deposition is primarily by charged leptons (electrons and positrons). The calculations show that the shower maximum occurs at an absorber depth of approximately $20 \mathrm{~cm}$ and the FWHM of the energy deposition is about $0.6 \mathrm{~cm}$. Integrating the deposited energy over the transverse plane at the shower maximum gives a deposited energy $0.335 \mathrm{GeV}$ $\mathrm{cm}^{2} / \mathrm{gm}$ per incident proton. Dividing by the minimum ionization energy loss in $\mathrm{Fe}, \mathrm{dE} / \mathrm{dx}, \min =1.45 \mathrm{MeV}-\mathrm{cm}^{2} / \mathrm{gm}$ then gives the equivalent flux of minimum ionizing particles (mips) crossing a transverse plane at the shower maximum, $335 / 1.45=231$ mips. The flux and mean energy of the shower particles per $300 \mathrm{GeV}$ proton at an absorber depth of $20 \mathrm{~cm}$ are given in Table 1. One can see from the Table that the total number of charged shower particles at the shower maximum is 155 per incident proton. On average then the charged shower particles are 231/155 $=1.49$ times minimum ionizing. 
Table I. The flux and mean energy of shower particles per $300 \mathrm{GeV}$ proton at depth of $18 \mathrm{~cm}$ in Fe.

\begin{tabular}{ccc}
\hline Particle & Flux per proton & Mean energy, MeV \\
\hline $\mathrm{e}^{-}$ & 84.6 & 73.0 \\
$\mathrm{e}^{+}$ & 55.4 & 108.0 \\
$\pi^{-}$ & 5.0 & 5,376 \\
$\pi^{+}$ & 5.7 & 6,440 \\
$\mathrm{~K}^{-}$ & 0.50 & 6,892 \\
$\mathrm{~K}^{+}$ & 0.59 & 8,501 \\
$\mathrm{p}$ & 3.4 & 35,795 \\
$\mathrm{n}$ & 17.0 & 1,970 \\
$\gamma$ & 1738 & 14.4 \\
All chg. & 155.2 & - \\
\hline
\end{tabular}

The equivalent flux of mips may be used to estimate the average induced electron charge collected from the ionization chamber per proton shower. A minimum ionizing particle produces 97 electron ion pairs per $\mathrm{cm}$ in Argon at one atmosphere pressure. The number of electron ion pairs produced in ten $0.5 \mathrm{~mm}$ gaps is then $(231 \times 97 \times 10 \times 0.05)=$ 11,203 . The average induced charge of electrons that are collected is one half of this value, or 5,602 e/proton shower at one atmosphere Ar.

\section{EXPERIMENTAL RESULTS}

\section{A. Electron drift velocity}

Measurements of the electron drift velocity in mixtures of Ar plus $1 \%, 1.5 \%, 2 \%$ and $3 \% \mathrm{~N}_{2}$ have been reported previously.[5] For these mixtures it was found that the saturation drift velocity and $\mathrm{E} / \mathrm{p}$ at saturation were increasing functions of $\mathrm{N}_{2}$ fraction. For most of the data in this report the $\operatorname{Ar}+2 \% \mathrm{~N}_{2}$ mixture was used with pressure varying from 250 to $1100 \mathrm{kPa}$ absolute. A constant electric field $6 \mathrm{kV} / \mathrm{cm}$ was applied to the ionization chamber by a battery power supply. For $\mathrm{Ar}+2 \% \mathrm{~N}_{2}$ and $\mathrm{E} / \mathrm{p}>1000 \mathrm{~V} / \mathrm{cm}-\mathrm{atm}$ the saturation drift velocity is $3.2 \mathrm{~cm} / \mu \mathrm{sec}$.

\section{B. Electronics bench tests and estimated signal amplitude}

Bench top measurements of the transfer function, rms noise voltage and equivalent noise charge (ENC) were carried out on the analog electronics used for the beam tests. With a $2.5 \mathrm{nsec}$ shaping time the transfer function for a Delta like charge injection with a base width of $8 \mathrm{nsec}$ was measured to be $0.45 \times 10^{-6} \mathrm{~V} / \mathrm{e}$. The ENC with a 3 meter cable and a $60 \mathrm{pF}$ load capacitance was measured to be $\sim 3,300 \mathrm{e}$. For an $18 \mathrm{nsec}$ wide triangular pulse and $2.5 \mathrm{nsec}$ shaping time the ballistic deficit was estimated to be between $(2.4 \sim 3.0)$. The transfer function and ballistic deficit may be combined with the estimate of collected charge to estimate the amplifier output signal at the shower maximum. The estimate is done for six atmospheres of $\mathrm{Ar}+2 \% \mathrm{~N}_{2}$ where most of our data were taken. Allowing for a measured $20 \%$ attenuation in the cable between the amplifier and data acquisition system and a average ballistic deficit of 2.75 , the mean amplitude of the signal expected for showers initiated by single protons is: $6 \mathrm{x}$ $11,200 \times 1 / 2 \times 0.45 \times 10^{-6} \times 0.8 / 2.75=4.4 \mathrm{mV}$.

\section{Pulse shapes and spectra}

\section{1) Typical waveforms}

Typical signals obtained at the maximum energy deposition of showers initiated by single $300 \mathrm{GeV}$ protons are given in Fig. 3 for four experimental conditions: (15a) two quadrants installed, (25b) single quadrant installed, (25n) single quadrant with series $27 \mathrm{pF}$ capacitor and (26f) single quadrant with series $10 \mathrm{pF}$ capacitor. In all cases the waveforms are averaged over 2,400 showers and the ionization chamber is filled with $600 \mathrm{kPa}\left(\mathrm{Ar}+2 \% \mathrm{~N}_{2}\right)$. The peak heights have been normalized to the single quadrant with no series capacitor (25b) and the respective normalization factors are given in the insert to Fig. 3. The amplitude of the single quadrant waveform is $4.2 \mathrm{mV}$ and corresponds to the estimated $4.4 \mathrm{mV}$ in Sec. IV.B. Notice in Figure 3 the broadening of the pulse by addition of a second quadrant and the narrowing by differentiation with a series capacitor. The width of the single quadrant pulse corresponds to a detector capacitance $\sim 60 \mathrm{pF}$, of the two quadrants $\sim 180 \mathrm{pF}$.

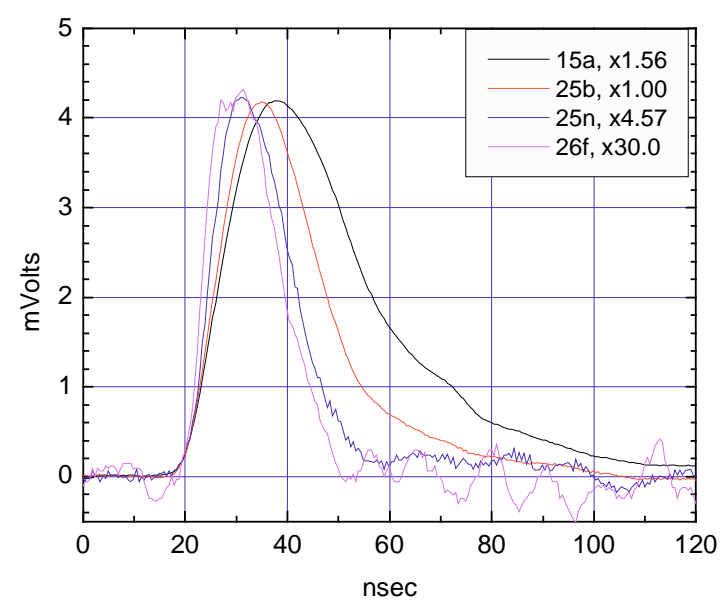

Fig. 3:Typical signals at the maximum energy deposition of showers initiated by single $300 \mathrm{GeV}$ protons.

\section{2) Pulse height and FWHM versus gas pressure}

Data were recorded varying the absolute pressure of $\mathrm{Ar}+$ $2 \% \mathrm{~N}_{2}$ from $250 \mathrm{kPa}$ to $1100 \mathrm{kPa}$. The peak amplitude and FWHM of these data are plotted versus pressure in Fig. 4. The electric field applied to the ionization chamber plates by the batteries was constant at $6,000 \mathrm{~V} / \mathrm{cm}$ so $\mathrm{E} / \mathrm{p}$ decreases with increasing pressure. The pulse amplitude increases linearly with pressure up to $600 \mathrm{kPa}$. The less than linear increase above $600 \mathrm{kPa}$ corresponds to the drift velocity falling below the saturation value $3.2 \mathrm{~cm} / \mu \mathrm{s}$ for $\mathrm{E} / \mathrm{p}<1,000 \mathrm{~V} / \mathrm{cm}-\mathrm{atm}$.[6]. In Fig. 4 one also sees that the FWHM is roughly constant at $21.5 \mathrm{~ns}$ for $\mathrm{E} / \mathrm{p}>1,000 \mathrm{~V} / \mathrm{cm}$-atm and then increases for $\mathrm{E} / \mathrm{p}<1,000 \mathrm{~V} / \mathrm{cm}-\mathrm{atm}$. 


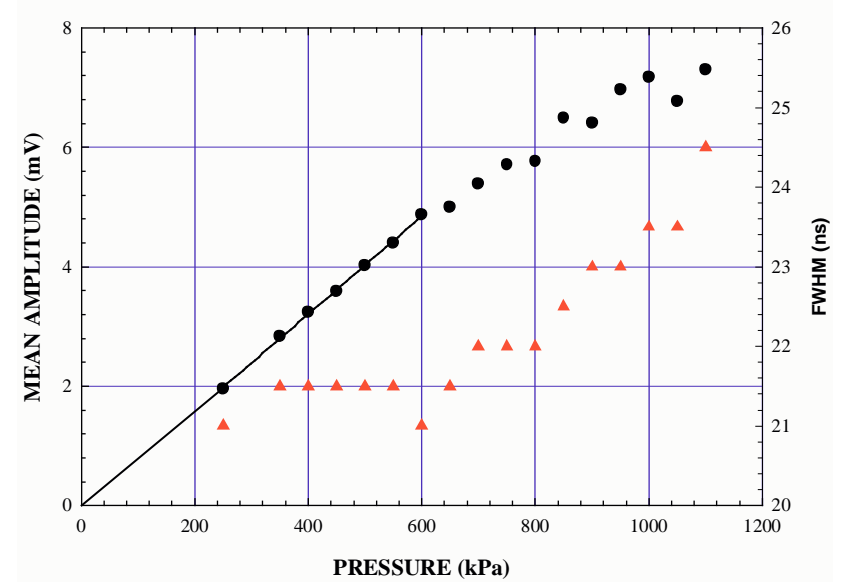

Fig. 4: Mean amplitude (left vertical axis) and FWHM (right vertical scale) versus pressure of $\mathrm{Ar}+2 \% \mathrm{~N}_{2}$. Electric field strength is held constant at $6,000 \mathrm{~V} / \mathrm{cm}$.

\section{IMPLICATIONS FOR 40MHZ OPERATION}

The data obtained so far have been obtained with widely spaced protons. However by synthesizing a pulse train from these data with pulses spaced $25 \mathrm{nsec}$ apart it can be demonstrated that the present system will allow measurement of events occurring at $40 \mathrm{MHz}$ repetition frequency. The problem reduces to demonstrating that the amplitudes of the individual pulses in a train can be determined without interference from the other pulses. The argument is made by synthesizing a pulse train with pulses of varying amplitude and then presenting an algorithm that allows the amplitudes of the individual pulses to be reconstructed from the summation waveform. If the full width of the individual pulses is less than the sum of the $25 n s e c$ bunch spacing and the peaking time, then algorithm would be especially straightforward - the amplitudes of the individual pulses would not interfere and can therefore be read off directly from the summation waveform with no de-convolution. When the full width of the pulses is greater than the sum of $25 \mathrm{nsec}$ and the peaking time the amplitudes interfere and can't be read directly from the summation waveform and de-convolution is necessary. However if the peaking time is less than the $25 \mathrm{nsec}$ bunch separation, which it is here, the de-convolution algorithm is very straightforward. The de-convolution makes use of the fact that the pulse shape of the charge sensitive amplifier is independent of amplitude. For the case under discussion, the amplitude of the leading pulse in the train is not influenced by the trailing pulses, its peak can be read directly from the summation waveform. The amplitude independent pulse shape of the charge sensitive amplifier is then fit to the peak of the leading pulse and the leading pulse subtracted from the pulse train. The second pulse then becomes the leading pulse, its amplitude read off, fitted with the amplitude independent pulse shape and subtracted. The process is repeated until the amplitude of each of the pulses in the train has been determined.
The procedure that has just been described is illustrated in Figs. 5, 6 and 7. Fig. 5(a) shows the mean waveform of 24,000 proton showers and Fig. 5(b) the mean waveform of the same showers taken 2,400 at a time. The tails of the pulse in Fig. 5(b) differ by random noise whereas the ten peak heights have statistically significant variations. The data were taken over a period of several hours and we speculate that the amplitude variation is due to a drift of the center of the proton beam relative to the detector. However the reason for this drift is not important for our discussion.
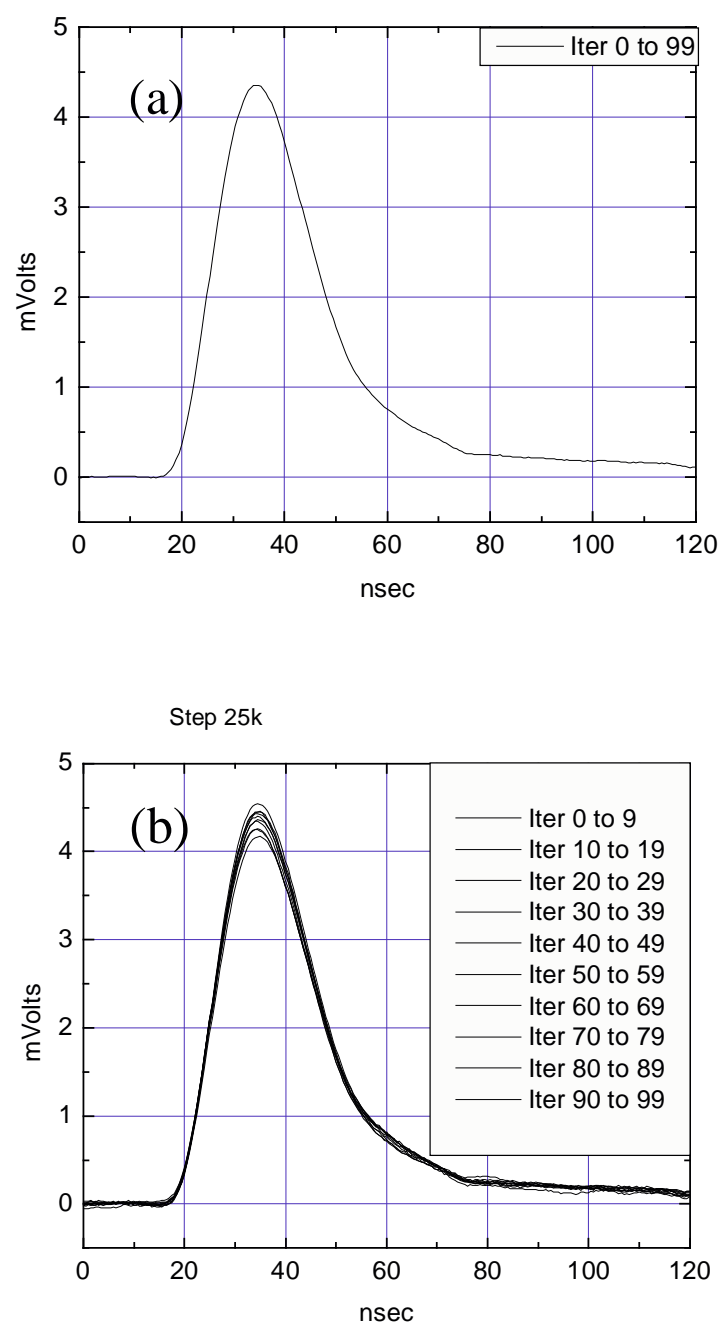

Fig. 5:(a) The mean waveform of 24,000 proton showers and (b) an overlay of the ten mean waveforms of 2,400 proton showers.

The summation of the ten waveforms in Fig. 5(b) displaced by $25 \mathrm{nsec}$ is shown in Fig. 6 . One can see the effect of pileup do to the pulse width extending beyond $25 \mathrm{nsec}$. Superimposing ten identical pulses shows the pileup saturates after three pulses. 


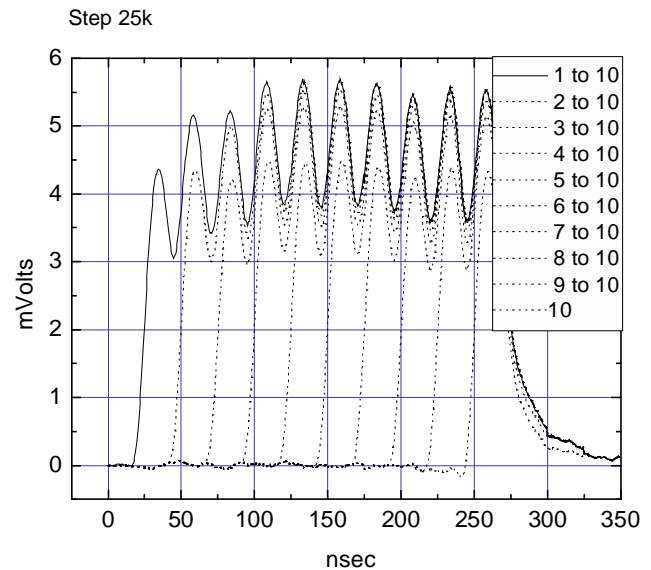

Fig. 6: A summation of the ten 2,400 event mean waveforms in Fig. 5(b) successively displaced by $25 \mathrm{nsec}$.

The shape of the 24,000 event waveform in Fig. 5(a) has been fit to the peak of the leading pulse in Fig. 6 and then subtracted to give a nine pulse train indicated by dashed lines in Fig. 6. The fitting and subtraction was then repeated until all ten pulses had been de-convolved. In Fig. 7 the peak raw peak heights of the ten individual waveforms in Fig. 5(b) have been plotted versus position in the ten-pulse train (open squares). The ten fitted peak heights obtained by the fitting and subtraction procedure are also plotted for comparison. One sees immediately in Fig. 7 that there is good correlation between the raw and fitted peak heights demonstrating the validity of the de-convolution. The raw and fitted peak heights for the first pulse agree exactly but differ for the trailing pulses owing to the effect of noise on the subtraction procedure. The raw and measured peak heights in Fig. 7 agree within $\pm 2 \%$. The creation of the pulse train by superposition in Fig. 6 does not exactly simulate a real train of proton showers separated by $25 \mathrm{nsec}$ because the electronic noise that is present gets added again with each pulse and accumulates up to five pulses since the superimposed pulses are $125 \mathrm{nsec}$ long. This causes the rms noise to increase by a factor of $5^{1 / 2}=2.24$ compared to a genuine pulse train.

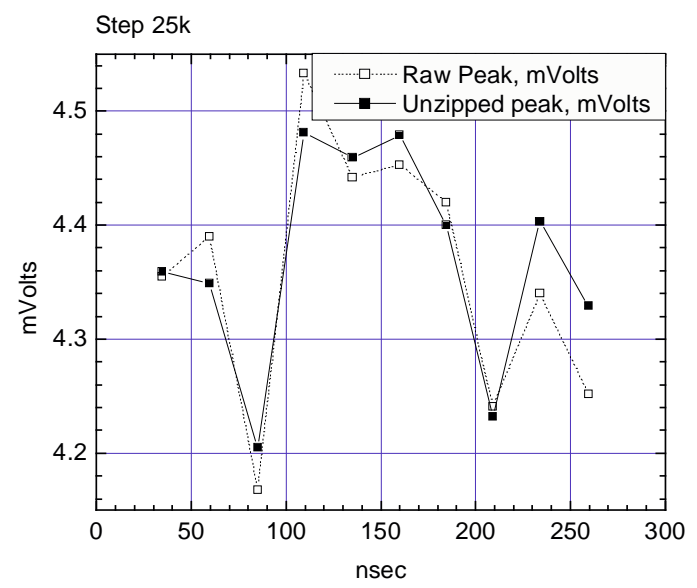

Fig. 7: Correlation of the raw peak heights of the ten 2,400 event waveforms with the peak heights obtained by the fitting and subtraction algorithm described in the text. The peak heights are plotted versus their position in the bunch train of Fig. 6.

\section{CONCLUSIONS}

In this paper we have given a status report on our work to develop a radiation hard $40 \mathrm{MHz}$ ionization chamber detector for use as storage ring operations tool for measuring and optimizing the bunch-by-bunch luminosity of the LHC. The first prototype was tested in a $450 \mathrm{GeV}$ proton beam and demonstrated the capability of the ionization chamber to detect hadronic/electromagnetic showers initiated by a single $450 \mathrm{GeV}$ proton. The second prototype has has been tested in a $300 \mathrm{GeV}$ proton beam and provided evidence that the ionization chamber approach to the luminosity optimization of the LHC can operate at the $40 \mathrm{MHz}$ bunch frequency. In the future we plan to test the ionization chamber in a $40 \mathrm{MHz}$ bunched beam when it is available.

\section{ACKNOWLEDGMENT}

We would like to thank Michael Hauschild, coordinator for SPS extracted beams and Per Grafstrom, liaison physicist for SPS H4 beam operations, for support and help in carrying out the experiments described in this report.

\section{REFERENCES}

[1] W.C. Turner, "Instrumentation for the Absorbers in the Low Beta Insertions of LHC", LBNL-42180, (13 Aug. 1998).

[2] W.C. Turner, P.S. Datte, P.F. Manfredi, J.E. Millaud, N.V. Mokhov, M. Placidi, V. Re and H. Schmickler, "Status Report on the Development of Instrumentation for Bunch by Bunch Measurement and Optimization of Luminosity in the LHC", Proc. of US LHC Collaboration Meeting on Accelerator Physics Experiments for Future Hadron Colliders, Brookhaven, 22-23 Feb. 2000.

[3] W.C. Turner, E.H. Hoyer and N.V. Mokhov, "Absorbers for the High Luminosity Insertions of the LHC," Proc. Of EPAC 98", Stockhom, 1998, pg. 368.

[4] W.C. Turner, M.T. Burks, P.S. Datte, P.F. Manfredi, J.E. Millaud, N.V. Mokhov, M. Placidi, L. Ratti, V. Re, H. Schmickler and V. Speziali, "Development of a detector for bunch by bunch measurement and optimization of luminosity in the LHC", Proc. of 8th Pisa Meeting on Advanced Detectors, Isola d'Elba, 21-27 May 2000.

[5] E. Gatti and P.F. Manfredi, "Signal Coupling from a Semiconductor Detector to a Cold Termination Resistance Preamplifier Through a Cable or a Delay Line Transformer", IEEE Trans. Nucl. Sci., Vol. NS-25, 66. (1978)

[6] J.-F. Beche, M.T. Burks, P.S. Datte, M. Haguenauer, P.F. Manfredi, J.E. Millaud, M. Placidi, L. Ratti, V. Re, V.J. Riot, H. Schmickler, V. Speziali and W. C. Turner, "An Ionization Chamber Shower Detector for the LHC Luminosity Monitor", LBNL-47438, 2000 IEEE Nuclear Science Symposium Conference Record, Lyon, Oct. 15-20, 2000, section 5, 136144. Accepted for publication in Transactions on Nuclear Science.

[7] P. Datte, P.F. Manfredi, J.E. Millaud, M. Placidi, L. Ratti, V. Speziali, G. Traversi and W.C. Turner, "Optimization of Signal Extraction and FrontEnd Design in a Fast Multigap Ionization Chamber", this conference.

[8] N.V. Mokhov, "MARS Code Developments, Benchmarking and Applications," Proc. ICRS-9 Intl. Conf. on Radiation Shielding", Tsukuba, Japan, Oct. 1999, and references therein.

[9] M. Burks, S. Trentalange, S. Margetis and H. Wieman, NIM A385, 519. (1997)

[10] P. Dreesen and G. Vismara, CERN-SPS/EA/78-4. (1978) 